\title{
Relevance and application of sortase and sortase-dependent proteins in lactic acid bacteria
}

\author{
Emma K. Call and Todd R. Klaenhammer* \\ Department of Food, Bioprocessing and Nutrition Sciences, North Carolina State University, Raleigh, NC, USA
}

\author{
Edited by: \\ Danilo Ercolini, Università degli Studi \\ di Napoli Federico II, Italy \\ Reviewed by: \\ Carmen Wacher, Universidad Nacional \\ Autónoma de México, Mexico \\ Diego Mora, Università degli Studi di \\ Milano, Italy \\ Marco Ventura, University of Parma, \\ Italy

\section{${ }^{*}$ Correspondence:} \\ Todd R. Klaenhammer, Department of \\ Food, Bioprocessing and Nutrition \\ Sciences, North Carolina State \\ University, 339 Schaub Hall, 400 Dan \\ Allen Dr., Box 7624, Raleigh, \\ NC 27695, USA. \\ e-mail: klaenhammer@ncsu.edu
}

\begin{abstract}
Lactic acid bacteria (LAB) are a diverse group of Gram-positive bacteria found in a vast array of environments including dairy products and the human gastrointestinal tract (GIT). In both niches, surface proteins play a crucial role in mediating interactions with the surrounding environment. The sortase enzyme is responsible for covalently coupling a subset of sortasedependent proteins (SDPs) to the cell wall of Gram-positive organisms through recognition of a conserved C-terminal LPXTG motif. Genomic sequencing of LAB and annotation has allowed for the identification of sortase and SDPs. Historically, sortase and SDPs were predominately investigated for their role in mediating pathogenesis. Identification of these proteins in $L A B$ has shed light on their important roles in mediating nutrient acquisition through proteinase $\mathrm{P}$ as well as positive probiotic attributes including adhesion, mucus barrier function, and immune signaling. Furthermore, sortase expression signals in LAB have been exploited as a means to develop oral vaccines targeted to the GIT. In this review, we examine the collection of studies which evaluate sortase and SDPs in select species of dairy-associated and health promoting LAB.
\end{abstract}

Keywords: probiotic, sortase, sortase-dependent protein, LPXTG, lactic acid bacteria, lactobacillus, lactococcus

\section{INTRODUCTION}

In Gram-positive bacteria, the cell wall is a crucial cellular component affecting a bacterium's fitness and survival. The cell wall is responsible for maintaining structural stability, providing a barrier to osmotic pressures, and facilitating interactions with the surrounding environment. Cell walls of Gram-positive bacteria are decorated with a vast array of macromolecular structures which facilitate these interactions. These structures include teichoic acids, lipoteichoic acids, exopolysaccharides, Surface (S)layer proteins, enzymes, and other cell surface proteins, such as adhesins and pili-like structures which are directly involved in host attachment (Marraffini et al., 2006; Weidenmaier and Peschel, 2008). The ecological niche of the microbes often dictates the mosaic-like surface display of macromolecules. The surface proteins of pathogenic microbes, such as internalin A in Listeria monocytogenes and protein A in Staphylococcus aureus, play a crucial role in establishing pathogenicity and infection (Mazmanian et al., 2000; Cabanes et al., 2002; Clancy et al., 2010). Alternately, in probiotic microbes, which confer health benefits upon the host, surface structures may play essential roles in eliciting these benefits.

Surface display is a twofold process composed of both protein targeting and protein attachment to the cell exterior. Protein targeting to the cell exterior is typically achieved through either the secretory (Sec) pathway or the twin-arginine translocation (TAT) pathway. The Sec pathway recognizes unfolded protein targets containing an N-terminal leader peptide, a hydrophobic core, and a C-terminal sequence that promotes binding of Sec machinery. Depending on the peptide sequence in the Cterminal region, the proteins are either exported out of the cell or N-terminally anchored in the membrane. Proteins that are
$\mathrm{N}$-terminally anchored in the membrane and processed by the Sec pathway represent a large proportion of membrane-anchored proteins in lactobacilli (Kleerebezem et al., 2010). Unlike the Sec pathway, the TAT pathway serves to transport folded protein to the cell's exterior. This pathway appears to be much more uncommon in species of lactic acid bacteria (LAB). To date this pathway has only been identified in Streptococcus thermophilus and not in lactococci or lactobacilli (Hols et al., 2005). Further association of these proteins targeted to the membrane and cell exterior can either be achieved through covalent linkages or non-covalent interactions. The non-covalent interactions which may allow for protein association with the cell wall following export by Sec or TAT machinery have been reviewed (Schaffer and Messner, 2005; Kleerebezem et al., 2010).

One class of proteins covalently associated with the peptidoglycan of the cell wall after Sec targeting are the LPXTG-anchored proteins. These proteins contain a C-terminal cell wall sorting signal with the sequence of amino acids leucine $(\mathrm{L})$, proline $(\mathrm{P}), \mathrm{X}$ (representing any amino acid substitution), threonine (T), and glycine $(G)$, and are linked to the cell wall by the housekeeping sortase, sortase A (SrtA). Successful linkage of sortase-dependent proteins (SDPs) to the cell wall is facilitated by the presence of not only the aforementioned LPXTG motif, but also a proceeding C-terminal hydrophobic region and a positively charged tail (Figure 1A). The $\mathrm{N}$-terminal region of SDPs contains a signal peptide. This signal peptide enables secretion of the sortase substrate by the Sec pathway, while the C-terminal charged tail anchors the substrate once it reaches the cell membrane. Anchoring in the cell membrane by the C-terminal tail brings the SDP and the sortase enzyme, also embedded in the cell membrane, into proximity so that it may carry out the transpeptidation reaction required for 
A
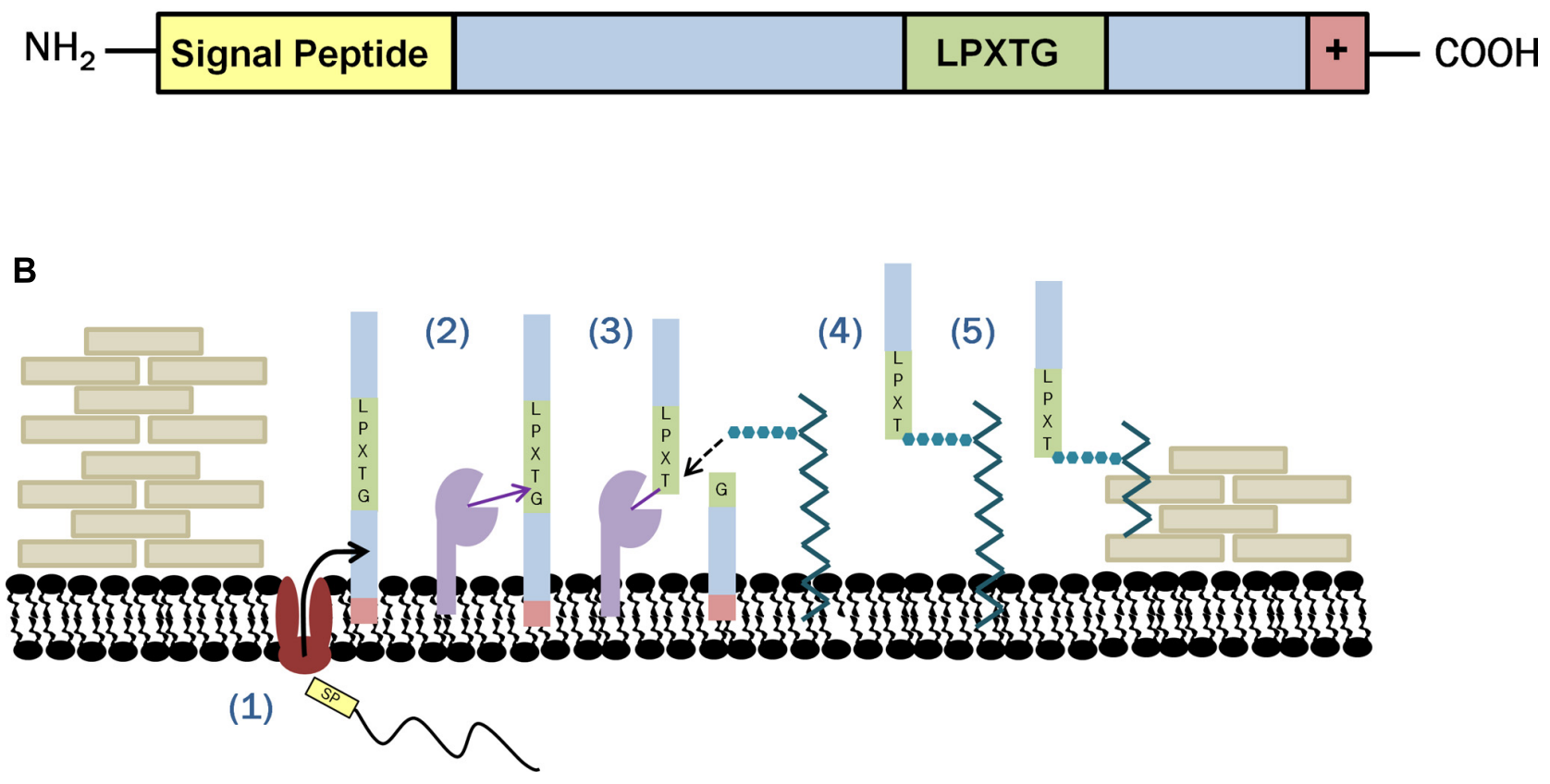

FIGURE 1 | Sortase anchoring in Gram-positive bacteria. (A) Sortase substrates are recognizable due to the presence of an $\mathrm{N}$-terminal signal peptide and a C-terminal LPXTG sorting signal followed by a series of hydrophobic and positively changed residues facilitating membrane anchoring. (B) Covalent linkage of sortase substrates to the cell wall is accomplished in a series of five steps: (1) Sec machinery recognizes the signal peptide on the sortase substrate and exports it to the cell's exterior. The substrate remains embedded in the membrane due to the presence of a hydrophobic region terminated by a charged tail. (2) Once sortase and the sortase substrate are in proximity, sortase cleaves the target between the glycine and threonine residues via a transpeptidase reaction. (3) Nucleophilic attachment by lipid II disassociates the sortase/SDP complex and (4) forms a lipid II intermediate through interaction with the pentapeptide cross bridge. (5) In the final stages of sortase anchoring, the sortase substrate is incorporated into the cell wall as part of normal cell wall construction (adapted from Hendrickx et al., 2011). cell wall anchoring. The first step in the transpeptidase reaction is the cleavage of the sortase substrate between the glycine and the threonine residue forming a sortase enzyme/SDP complex. The resulting thioester acyl bond between these two proteins is then subjected to nucleophilic attack and subsequent linkage to lipid II. Although lipid II is composed of both the peptidoglycan precursors, $N$-acetylglucosamine and $N$-acetylmuramic acid, as well as the pentapeptide peptidoglycan cross bridge, SDPs have been shown to link specifically to the pentapeptide (Maresso and Schneewind, 2008; Kleerebezem et al., 2010; Spirig et al., 2011). Once linked to the cross bridge, SDPs are incorporated into the cell wall with lipid II as it is translocated to the outer surface of the cell (Figure 1B).

Class A sortases which couple SDPs to the cell wall are the most well characterized; however, other classes of sortase enzymes have been identified. Class B sortases have been implicated in heme acquisition from the host, while class D, E, and F sortases have been identified and functionally analyzed to a lesser extent (Spirig et al., 2011). Class C sortases are better studied and play a critical role in pilus assembly. Class $\mathrm{C}$ sortases are transpeptidases, like class A sortases; however, they recognize a different sorting motif of (Isoleucine [I]/L)(P/Alanine [A]) XTG (Hendrickx et al., 2011). Functionally, class $\mathrm{C}$ sortases catalyze linkages between the Spa proteins to elongate the pilus shaft. Once elongation is complete, the pilus is anchored to the cell wall by either the class $\mathrm{C}$ sortase itself or by a class A sortase (Spirig et al., 2011). Similar to proteins coupled to the cell wall by class A sortases, the pili formed by class $\mathrm{C}$ sortases represent another mechanism of bacterial interaction with the environment. Pili are responsible for adherence to epithelial cells and extracellular matrix proteins, interaction with the host immune system, as well as biofilm formation (Danne and Dramsi, 2012).

Sortase proteins have been most extensively studied in the context of pathogens. SrtA was first identified in S. aureus where it is responsible for coupling between 18 and 22 substrates to the cell wall depending on the species (Marraffini et al., 2006). In $S$. aureus, as well as in L. monocytogenes and other Gram-positive pathogens, the deletion of the gene encoding the sortase enzyme showed attenuated virulence in animal models (Mazmanian et al., 2000; Bierne et al., 2002; Garandeau, 2002). This decrease in infective capability is attributed to the loss of adhesins, internalins, clumping factors, or host evasion molecules such as protein A from the cell surface of $S$. aureus. Not surprisingly, the product of sortase $\mathrm{C}$ action, the pilus, has also been implicated in pathogenicity through adhesion and host cell invasion in, but not limited to, Corynebacterium diphtheriae, Streptococcus pneumoniae, Streptococcus pyogenes, and Actinomyces naeslundii (Ellen et al., 1978; Ton-That and Schneewind, 2003; Mora et al., 2005; Barocchi et al., 2006; Gaspar and Ton-That, 2006; Telford et al., 2006; Mishra et al., 2007). Taken together, these findings were suggestive that 
sortase inhibition could function as an anti-infective therapy as thoroughly reviewed by Maresso and Schneewind (2008).

Sortase enzymes are found in all Gram-positive microbes, including food grade and health-relevant microbes of the LAB. Members of the LAB have a history of safe use and consumption of these microbes has been associated with health benefits, including competitive inhibition of pathogens, maintenance of epithelial barrier function, and a reduction in the symptoms of irritable bowel syndrome (Ventura et al., 2009). Nonetheless, in many cases the mechanisms associated with these effects are unknown. The sortase enzyme and SDPs in LAB are of interest in delineating the molecular mechanisms of host-bacterial interaction. While SrtA enzymes have been identified in a handful of LAB members, the sortase C enzyme has only been functionally characterized in Lactobacillus rhamnosus GG (Kankainen et al., 2009). Furthermore, the sortase cell wall anchoring machinery in LAB has been explored in the development of vaccines which could be administered orally as strains generally recognized as safe (GRAS).

\section{COMPARATIVE ANALYSIS OF SORTASE IN LAB}

As previously stated, the sortase protein is ubiquitous among Gram-positive bacteria, and members of the LAB family are no exception. Sequencing of various LAB genomes has allowed for the identification of bacterial genes, including those that code for sortase. In 2002, a collaborative effort between the Department of Energy - Joint Genome Institute and the scientists of the Lactic Acid Bacteria Genome Consortium announced an elaborate sequencing project which aimed to make LAB genomes available to the public. Four years after the announcement of the initiative, 18 genomes of LAB were publically available (Makarova et al., 2006). Approximately a decade later, over 26 LAB genomes are available (Zhou et al., 2010). Encompassed in this collection of LAB are probiotic strains which include Lactobacillus acidophilus, Lactobacillus gasseri, and Lactobacillus plantarum. The genome assemblies have shed light on the presence of cell surface-associated structures which are suggested to modulate the microbe-host response.

The role of sortase enzymes in the attachment of proteins to the cell wall makes it an attractive target for genome mining of structures involved in bacterial-host interactions. Genes encoding housekeeping sortase enzymes ( $s r t A$ ) have been identified in the genomes of LAB including L. acidophilus (Buck et al., 2005), Lactobacillus salivarius UCC118 (van Pijkeren et al., 2006), Lactobacillus johnsonii NCC533 (Denou et al., 2008), L. rhamnosus GG (Kankainen et al., 2009), Lactococcus lactis IL1403 (Dieye et al., 2010), Lactobacillus casei BL23 (Munoz-Provencio et al., 2012), L. plantarum (Remus et al., 2013), and Lactobacillus crispatus ST1 (Edelman et al., 2012). Additionally, genes encoding class C sortase proteins $(s r t C)$ have been identified in both L. rhamnosus GG (Kankainen et al., 2009) and L. casei BL23 (Munoz-Provencio et al., 2012). The genomic context of the $s r t A$ locus varies widely among species (Figure 2A); however, srtC genes have been found to cluster with their targets (spa genes) as was observed in both $L$. rhamnosus GG (Figure 2B) and L. casei BL23 (Munoz-Provencio et al., 2012). An unrooted phylogenetic tree shows the relationships between the sortase protein sequences identified in the aforementioned species of LAB as well as those present in other LAB species including L. lactis subsp. cremoris MG1463 and S. thermophilus
LMD-9 (Figure 3). As expected based on differences in target proteins, distinct clusters formed indicating divergence in amino acid sequences of the housekeeping sortase, SrtA, and the pilin sortase, SrtC. Additionally, the SrtA proteins from group A members of the acidophilus complex cluster independently from those of the group B acidophilus complex, and those LAB (i.e., L. lactis and $S$. thermophilus) whose 16s rRNA are divergent from those of lactobacilli also possess SrtA enzymes which cluster independently.

Further analysis of the genome content of these species allows identification of those substrates that sortase covalently links to the cell wall. SDPs can be identified based on the presence of a C-terminal LPXTG motif as well as an N-terminal signal peptide followed by a series of hydrophobic residues, as previously discussed. SDPs were first identified in sequenced genomes using from a hidden Markov model which predicted 732 sortase targets in 49 different prokaryotes (Boekhorst et al., 2005). Years later, those LPXTG containing proteins found in prokaryotic LAB were compiled and combined with other cell wall anchored and secreted structures to form the LAB secretome database ${ }^{1}$ (Zhou et al., 2010). This database currently contains 26 LAB and is publically available to aid in the identification of SDPs as well as other secreted structures. Although the software is capable of identifying SDPs, it is unable to distinguish between those SDPs which are pseudo or truncated genes, and those SDPs which are fully functional genes containing the three domains described above. The presence and of the signal peptide and other protein domains can be determined using InterProScan ${ }^{2}$, while the functionality and cleavage site within the signal peptide can be determined using SignalP 4.0 software $^{3}$. A combination of these tools and a survey of the current literature was used to develop a table summarizing the prevalence of sortase enzymes, SDPs, and the functionality of SDPs in some common LAB (Table 1).

\section{FUNCTIONAL ANALYSIS OF THE HOUSEKEEPING SORTASE AND SORTASE-DEPENDENT PROTEINS (SDPS) IN LAB}

Prior to genome mining for SDPs, Roos and Jonsson (2002) first described the function role of a protein in the SDP family in Lactobacillus reuteri 1063 . The previously uncharacterized putative cell surface protein (NCBI reference sequence: AF120104) contained repeat regions homologous to mucus-binding (mub) domains and a C-terminal sortase recognition LPQTG motif. Furthermore, the protein contained an N-terminal secretion signal consistent with the conserved structure of functional sortase targets. The protein was called "Mub" and recombinant forms of the protein showed adherence to mucin derived from different animal species (Roos and Jonsson, 2002). Although the publication did not identify a sortase protein itself, the genome sequence of a different strain of L. reuteri (DSM 20016) with an available genome indicated the presence of a sortase enzyme (NCBI reference sequence: YP_001270843.1). This study asserted the first suggestion of an adhesive protein in a Lactobacillus species, and furthermore, the first suggestion that LPXTG-anchored motifs may play a role in mucus-binding in the gastrointestinal tract (GIT) of the host.

\footnotetext{
${ }^{1}$ http://www.cmbi.ru.nl/lab_secretome/index.php

${ }^{2}$ http://www.ebi.ac.uk/Tools/pfa/iprscan/

${ }^{3}$ http://www.cbs.dtu.dk/services/SignalP/
} 
A

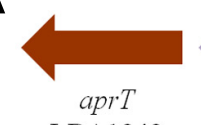

LBA 1242

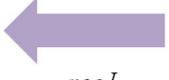

rec J

LBA1243

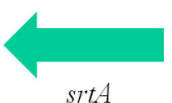

LBA1244

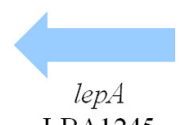

LBA1245

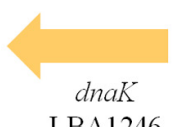

LBA1246

\section{L. acidophilus NCFM}

\section{L. plantarum WCFS1}

\section{L. rhamnosus GG}

\section{L. lactis subsp. lactis IL1403}

\section{L. rhamnosus GG $\operatorname{srt}_{1}$}

\section{L. rhamnosus GG srtC $_{2}$}

B

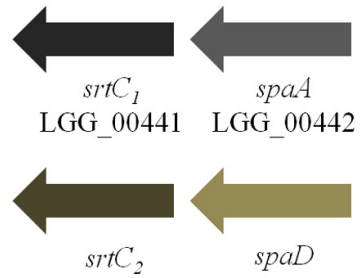

LGG 02369 LGG 00470

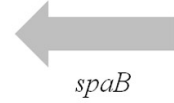

LGG 00443

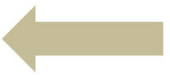

spaE

LGG 00471

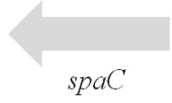

LGG 00444

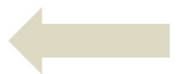

spaF

LGG 00472
FIGURE 2 | Genomic context of genes encoding sortases in LAB. (A) Genomic context of sortase A (green arrow) as found in L. acidophilus NCFM, L. plantarum WCFS1, L. rhamnosus GG, and L. lactis subsp. lactis IL1403 vary greatly. The sortase in $L$. lactis is not yet annotated as such; however, the gene annotated $y / c C$ was identified to be the putative sortase enzyme by
Dieye et al. (2010). These species were chosen to represent species from members of the acidophilus complex, other probiotic lactobacilli, and food-associated LAB. (B) Unlike sortase A whose targets are dispersed throughout the genome, sortase $\mathrm{C}$ enzymes cluster with their pili-subunit targets (adapted from Kankainen et al., 2009)
Similar adhesion factors were identified in L. acidophilus NCFM after its genome sequence became available in 2005 (Altermann et al., 2005). Three proteins, LBA1633, LBA1634, and LBA1392, were identified for their putative adhesive capacity based on their sequence homology with R28 protein from S. pyogenes (Buck et al., 2005). In S. pyogenes, R28 plays a role in binding to a cervical epithelial cell line (Stalhammar-Carlemalm et al., 1999). Additionally, the L. acidophilus protein, LBA1392, was found to have $25 \%$ protein homology to the Mub identified in $L$. reuteri (Buck et al., 2005). Interestingly, all three of these proteins contained a C-terminal LPXTG sorting signal. The presence of the LPXTG signal in these three putative Mubs as well as in nine other open reading frames in the L. acidophilus genome were suggestive of the presence of a sortase protein identified as LBA1244. Deletion and functional analysis of the sortase linked Mub, LBA1392, showed significant impairment in adherence to humans Caco-2 epithelial cells, but this observation was not reproducible for LBA1633 or LBA1634 (Buck et al., 2005). The nine other predicted sortase targets remained uninvestigated in this study.
The aforementioned studies in $L$. reuteri and L. acidophilus NCFM implicated surface proteins in specific adhesive roles of probiotic bacteria in the GIT and directed attention to the sortase protein and SDPs as an important mediator of this positive probiotic attribute. Further comparative analysis of cell surface structures associated with probiotic bacteria revealed that LPXTGanchored targets could be found in common probiotic species including L. plantarum WCFS1, L. johnsonii NCC 533, Lactobacillus sakei $23 \mathrm{~K}$, and L. salivarius UCC118 (Table 1). Furthermore, the number of targets present varied from species to species, with the highest number found in L. plantarum WCFS1 (Kleerebezem etal., 2010). While the role of sortase and SDPs in $L$. plantarum was evaluated at a later time (Remus et al., 2013), the initial focus was placed on determining the functional role of sortase and SDPs in Lactobacillus salivarius UCC118. Publication of its genome revealed the presence of ten SDPs consisting of four intact targets and six pseudogenes (Claesson et al., 2006; van Pijkeren et al., 2006).

With the genome sequence available, functional analysis of sortase and SDPs in L. salivarius UCC118 were greatly expedited. 


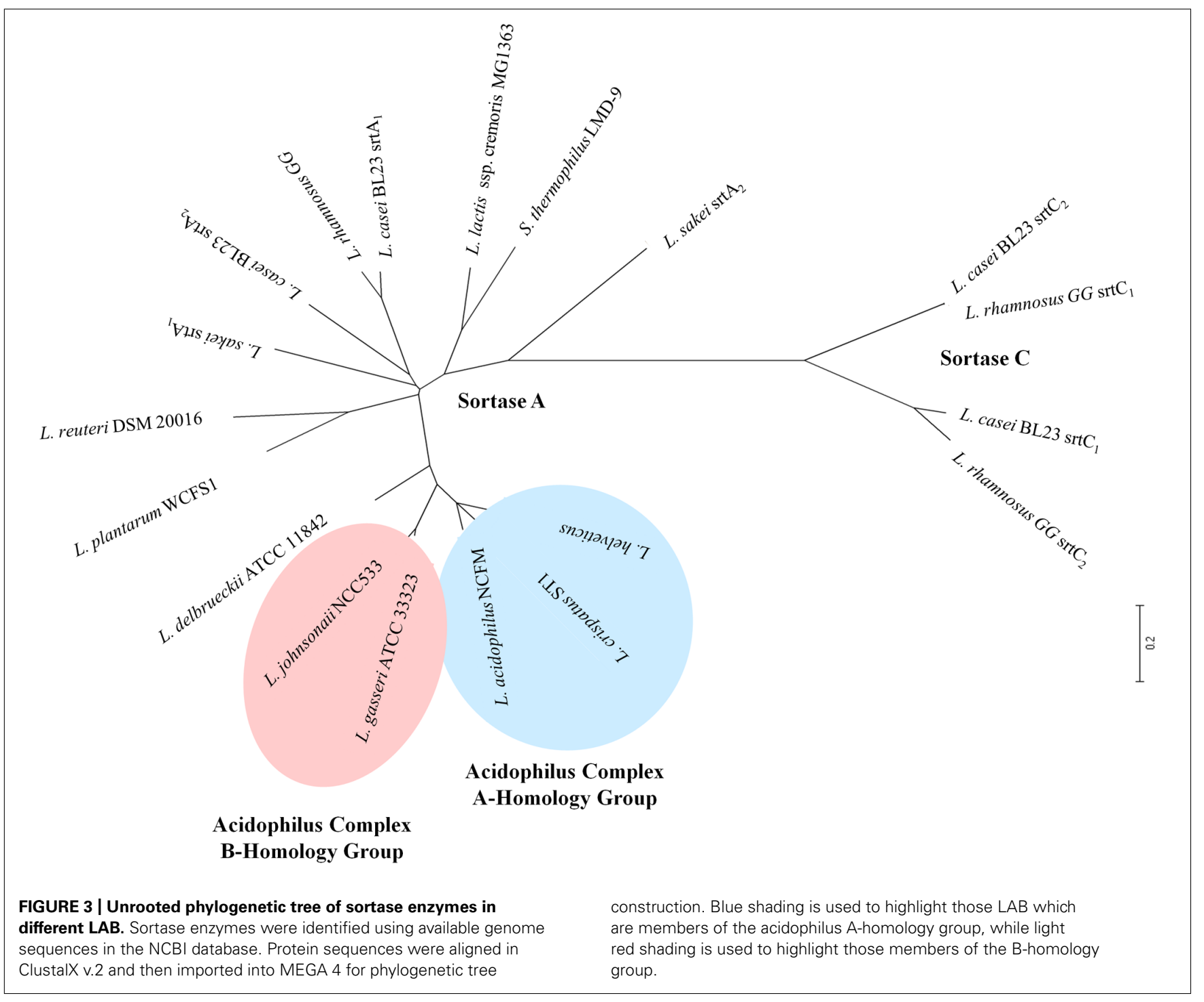

The sortase gene, identified as LSL_1606, was deleted while the four functional SDPs were insertionally inactivated (van Pijkeren et al., 2006). In two separate in vitro adhesion assays, one using Caco-2 and one using HT-29 adenocarcinoma epithelial cells, the sortase-deficient strain showed significant decreases in adhesion. One of the SDPs, named LspA (LSL_0311), was shown to contribute to this phenotype, while two others ( $\mathrm{LspB}$ and $\mathrm{LspD}$ ) did not appear to significantly reduce adhesion in these model systems. Not surprisingly, the SDP LspA contained a series of mucus-binding domain as were previously described as involved in adhesion in both L. reuteri and L. acidophilus NCFM (van Pijkeren et al., 2006). Current annotations of this protein describe it as Mbp2 since Lsp is used to denote lipoproteins structures unrelated to sortase.

A collection of SDPs from other probiotic microbes, including the mannose-specific adhesin (msa) in L. plantarum 299v (Gross et al., 2008) and the Lactobacillus epithelial adhesin (LEA) isolated from L. casei BL23 (Edelman et al., 2012) have been shown to contribute to bacterial adhesion. In L. casei BL23, twenty three
SDPs were predicted; however, none were specifically targeted for investigation. Alternately, each of the four sortases genes (two $s r t A$ and two $s r t C$ ) were inactivated and the adhesion phenotype examined. In this study, inactivation of both SrtA enzymes was required to functionally impact binding to colonic epithelial cell lines, while inactivation of either of the class $\mathrm{C}$ sortases did not impact adhesion (Edelman et al., 2012). The latter finding was unexpected since in L. rhamnosus GG this gene is essential in constructing pili which directly contribute to epithelial cell adhesion (Kankainen et al., 2009). It is unclear which of the twenty three predicted SDPs plays a specific role in the adhesion phenotype as none share homology with Mubs found in L. acidophilus or L. reuteri (Edelman et al., 2012). However, one SDP (LCABL_23040) shares homology with a mucus-binding factor (MBF) in L. rhamnosus GG and may provide insight into SDPs mediating adhesion in L. casei BL23 (Munoz-Provencio et al., 2012).

Recent interest in sortase and SDPs has begun to focus on the role of these proteins in the immunomodulatory capacity of probiotic bacteria. These bacteria have the capacity to 
Table 1 | Comparative genomic analysis of sortase-dependent proteins in select species of LAB.

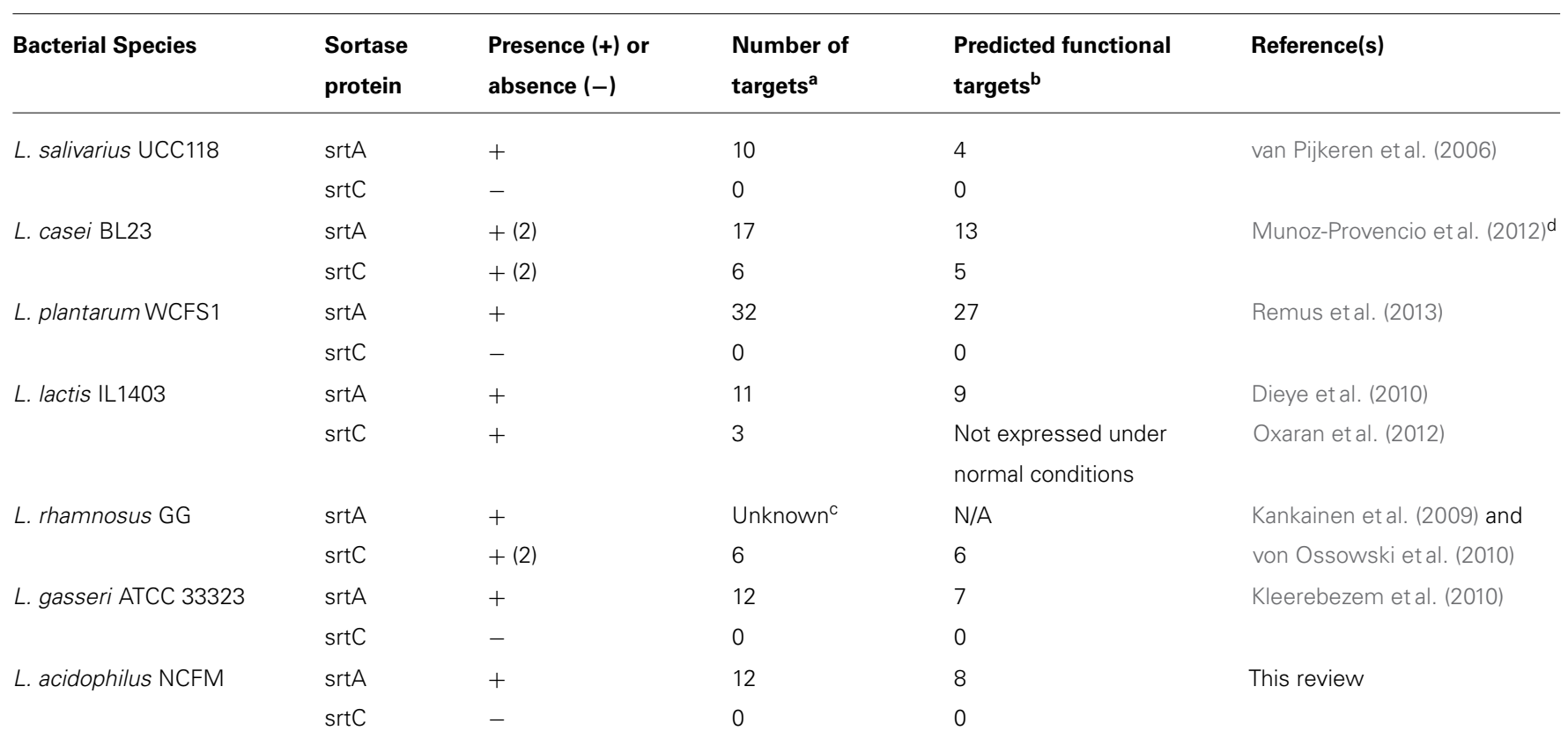

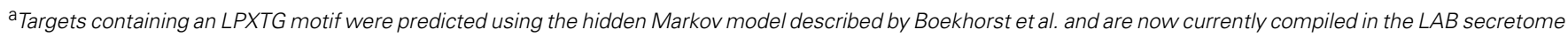
database (Boekhorst etal., 2005; Zhou etal., 2010).

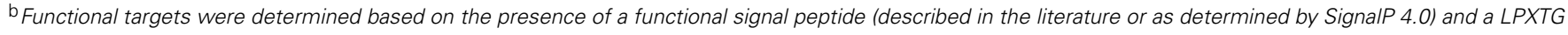
or LPXTG-like motif.

${ }^{C}$ L. rhamnosus GG is not a part of the LAB secretome database developed by Zhou etal., 2010.

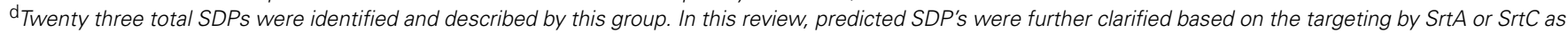
well as their predicted functionality based on the parameters above.

influence immune signaling of the colonic epithelium directly and through modulation of NF- $\kappa \mathrm{B}$ signaling pathways. As an example, L. rhamnosus GG and L. plantarum BFE have been shown to enhance innate immune signals by increasing the expression of Toll-like receptors (TLRs) in HT-29 cells (Pinto et al., 2009). Additionally, antagonists of the potent pro-inflammatory transcriptional regulator, NF- $\kappa \mathrm{B}$, were up-regulated after one hour exposure of Caco-2 cells to L. acidophilus NCFM based on microarray analysis (O'Flaherty and Klaenhammer, 2012). This same technique was used to investigate transcriptional responses of Caco-2 cells exposed to both the sortase-deficient mutant of L. salivarius UCC118 and the wild type (O'Callaghan etal., 2012). Although the wild type strain showed down regulation of the NF- $\mathrm{B}$ antagonist as well as induction of some innate immune regulators such as chemokines, the immune signaling pathways did not appear to be different after exposure to $\Delta s r t A$ L. salivarius UCC118. However, epithelial mucin genes were significantly down-regulated after exposure to $\triangle$ srtA L. salivarius UCC118 (O'Callaghan et al., 2012). Mucin is important in the colonic epithelium for maintaining lubrication and barrier functionality of the GIT as well as preventing pathogen penetration into epithelial cells of the GIT (Shirazi et al., 2000; Moran et al., 2011). Down-regulation of the mucin genes after exposure to L. salivarius UCC118 lacking sortase and thus SDPs, implicate SDPs in stimulating mucin production to maintain barrier function as well as in adhesion, as previously described (van Pijkeren et al., 2006).
The role of sortase in non-pathogenic species has been focused, but not limited to, probiotic LAB. Initial studies using lactococci as a vaccine carrier suggested sortase machinery was functional due to the ability to display known sortase-anchored proteins from other species (e.g., M6 from S. pyogenes) on the surface of L. lactis ssp. cremoris (Norton et al., 1996; Piard et al., 1997). Additionally, amino acid sequence comparison of the L. lactis ssp. cremoris genes including the sex-factor aggregation gene $(\operatorname{clu} A)$ and proteinase $\mathrm{P}$ (PrtP) showed regions of homology to the LPXTG domains of cell wall-anchored proteins (Vos et al., 1989; Godon et al., 1995). This provided evidence for localization of PrtP at the cell wall and its display outside the cell envelope in a location important for nutrient acquisition in a dairy environment (Vos et al., 1989). Further investigation into sortase machinery in the industry-relevant strains has been accomplished in L. lactis ssp. lactis IL1403. This specific L. lactis has two sortase genes, srtA and srtC. The srtC gene is only expressed at low levels and does not appear to build functional pili under normal growth conditions (Oxaran et al., 2012). In contrast, SrtA couples at least five proteins to the cell wall of L. lactis ssp. lactis IL1403, some of which were shown to contain mucus-binding domains homologous to those present in some lactobacilli. This finding is suggestive of potential binding capacity to cellular components of the human GIT although this has not been demonstrated experimentally. In addition, PrtP is not found in this particular species although it can be found it L. lactis ssp. cremoris MG1363 (Dieye et al., 2010). 
Variation in the sortase gene in species of $S$. thermophilus can also be observed. For example, the genome of $S$. thermophilus LMD-9 appears to encode an intact sortase, while the genomes of S. thermophilus CNRZ1066 and S. thermophilus LMG 13811 both harbor truncated enzymes (Goh et al., 2011). Furthermore, the latter two species do not contain genes encoding SDPs (Bolotin et al., 2004), while S. thermophilus LMD-9 potentially contains three SDPs, as predicted by the LAB secretome database (Zhou et al., 2010; Goh et al., 2011). In the case of S. thermophilus CNRZ1066 and S. thermophilus LMG 13811 this further substantiates the hypothesis of genomic decay during adaptation to milk and loss of gene features, including cell surface proteins, shown to contribute to virulence in related streptococcal pathogens (Bolotin et al., 2004).

This collection of studies represents the state of functional analysis of the housekeeping SrtA and its targets in probiotic species of lactobacilli as well as the limited investigation of this enzyme in food-associated L. lactis and S. thermophilus. While the studies published on SrtA in probiotic lactobacilli are not exhaustive, they suggest an important role for this enzyme and SDPs in adhesion to the intestinal mucosa of the host. Moreover, the functional role of sortase in adhesion is predicted to be mediated through its role in linking mucus-binding proteins or similar protein structures, such as the MBF found in L. rhamnosus GG (Munoz-Provencio et al., 2012), to the cell wall. Additionally, although not investigated in great detail at this point in time, the adhesive capacity of different probiotic strains to mucus and the GIT may function to allow interactions with the local immune system in the GIT. Beyond investigating gene expression of epithelial cell lines such as Caco- 2 cells after probiotic exposure, it may be prudent to explore the responses of dendritic cells (DCs) to such treatment. DCs are resident immune cells in the GIT with the capacity to sample antigens and signal the immune system through cytokines. This approach to investigating immune stimulation by probiotic bacteria has been employed with regard to a sortase-deficient mutant of L. plantarum WCFS1, although significant changes in the amounts of anti-inflammatory IL-10 and pro-inflammatory IL-12p70 were not detectable when DCs were cultured with the individual strains (Remus etal., 2013). Studies such as these with other strains, both wild type and sortase-deficient, will help unravel the mechanisms behind probiotic functionality. Namely, as suggested by O'Callaghan et al. (2012) the combined adhesive capacity provided by some SDPs and the immune stimulation induced by probiotics, acting together, may condition the GIT for potential pathogen exposure.

\section{THE PILI SORTASE: CHARACTERIZATION OF SrtC IN L. rhamnosus GG}

Like the housekeeping sortase, originally discovered in the Grampositive pathogen $S$. aureus, the sortase responsible for pili assembly, sortase $\mathrm{C}$, was first described in the pathogen $C$. diphtheriae (Ton-That and Schneewind, 2003). It was shown to play a key role in assembling subunits, namely SpaA and SpaC, to form the pili found to protrude from the surface of $C$. diphtheriae. Pili are filamentous structures, approximately 1-2 $\mu \mathrm{m}$ in length, and usually numerous in their display on the bacterial surface. Since their discovery in C. diphtheriae, pili have been described in many more
Gram-positive pathogens as key components involved in host tissue colonization; however, until 2009 these structures remained undiscovered in commensal lactobacilli (Kankainen et al., 2009).

In 2009, the presence of mucus-binding pili displayed on the surface of L. rhamnosus GG was described (Kankainen et al., 2009). L. rhamnosus GG is a probiotic bacterial strain which has been used for over two decades. Additionally, L. rhamnosus GG shows exemplary ability to adhere to Caco-2 cells as compared to other probiotic strains (Jacobsen et al., 1999). The genome sequence of L. rhamnosus GG revealed two potential clusters of pilus-encoding genes in tandem with a srtC gene (Figure 2B). The first cluster identified contained genes for spaA (LGG_00442), spaB (LGG_00443), and spaC (LGG_00444) clustered with $s r t C_{1}$ (LGG_00441), while the second cluster contained genes for spaD (LGG_02370), spaE (LGG_02371), and spaF (LGG_02372) clustered with srtC2 (LGG_02369; Kankainen et al., 2009). Furthermore, demonstration of the expression and presence of pilin-like structures on the surface of L. rhamnosus GG was accomplished using immunogold transmission electron microscopy, as was first described by Ton-That and Schneewind (2003) in C. diphtheriae. Double labeling, first with primary antibodies to the SpaC subunit of the pili and subsequently with a secondary antibody containing gold nanoparticles, allows the pili to be detected under transmission microscopy. Remarkably, the pili were not only identifiable, but they were relatively numerous at approximately 10-50 pili per cell (Kankainen et al., 2009).

The spa genes found in L. rhamnosus GG have been further characterized with regard to their function in assembling pili and in their ability to adhere to mucin. The protein product of the first gene following $s r t C_{1}$, the SpaA subunit, forms the backbone of the pili in L. rhamnosus GG. SpaB is found at the base of the pilin structure and is likely attached to the cell wall through the action of the SrtA enzyme, which is also encoded in the genome. Finally, SpaC can be found flanking the pilin shaft. SpaB, and to some extent SpaC, contribute to the adhesive capacity of L. rhamnosus GG to mucin through different mechanisms (Reunanen et al., 2012). Insertional inactivation of the spaC gene essentially abolishes binding to human intestinal mucus, and expression and further purification of this pilus subunit from $E$. coli showed significant binding to immobilized human intestinal mucus. The SpaB protein showed even the greatest degree of binding to human intestinal mucus which was attributed to its net positive charge facilitating binding with negative residues present in human mucus (von Ossowski et al., 2010).

To date, pili have not been functionally identified in other species of food-adapted or probiotic lactobacilli. Gene clusters with homologous structures to those found in L. rhamnosus GG have been described in L. casei BL23. In L. casei BL23, the gene cluster encoding spaA, spaB, spaC, and a class C sortase appear to be genetically intact; however, functional pili on the surface of L. casei BL23 has not been reported (Munoz-Provencio et al., 2012). Furthermore, the gene cluster encoding spaD, spaE, and $s p a F$ appears to be present, but with truncations in $s p a E$ and $s p a F$. Recently, a gene encoding a sortase $\mathrm{C}$ homolog flanked by three genes with LPXTG motifs was identified in L. lactis IL1403. The expression of the genes and the formation of pili could not be detected under normal growth conditions; however, cloning and 
overexpression of the gene cluster under a high copy lactococcal promoter led to pili display (Oxaran et al., 2012).

\section{LPXTG MOTIF AND BIOTHERAPEUTIC APPLICATION IN LACTIC ACID BACTERIA}

The conserved C-terminal anchor motif recognized by sortase in Gram-positive microbes has been suggested as a means of antigen display in vaccine development (Norton et al., 1996; BermudezHumaran et al., 2003; Cortes-Perez et al., 2003, 2005; Fredriksen etal., 2010; Kajikawa etal., 2011). Specifically, the use of the LPXTG motif has been investigated for in vitro vaccine delivery using food grade and probiotic lactobacilli as the presentation vector for the antigen. Unlike vaccine delivery vehicles which rely on attenuated strains of pathogenic bacteria, food grade LAB and notably probiotic lactobacilli present an alternative delivery vehicle as they have a safe history of use in foods and dietary supplements, are GRAS, and are able to survive passage through the GIT for vaccine delivery to the mucosal immune system. In addition, LAB have been shown to also have Sec and C-terminal cell wall anchoring machinery which can be exploited for antigen immobilization. To date, LAB and sortase-mediated cell wall anchoring have been explored in the display of potential vaccine antigens including tetanus toxin fragment C (TTFC; Norton et al., 1996), human papillomavirus (HPV) type 16 E7 antigen (BermudezHumaran et al., 2003; Cortes-Perez et al., 2003, 2005), the oncofetal antigen (Fredriksen et al., 2010), and Salmonella enterica serovar typhimurium flagellin (FliC; Kajikawa et al., 2011).

The functionality of sortase-mediated cell wall localization in $\mathrm{LAB}$ was demonstrated through display of the M6 protein, a LPXTG-anchored virulence factor of S. pyogenes, in L. lactis. The M6 protein was also successfully displayed on the cell wall of other LAB including L. fermentum LEM83, L. sakei 23K, and S. thermophilus CNRZ302. This was achieved through cloning of the gene encoding the M6 protein (emm6) into the aforementioned $\mathrm{LAB}$ and then examining the distribution of the M6 protein using Western blot analysis. The authors were able to demonstrate the conserved nature of sortase anchoring among Gram-positive organisms; however, they noted differences in anchoring efficiency between different LAB. For example, M6 protein could be detected in the supernatant from the cocci, while it was not readily detected in the supernatant collected from the rod-shaped lactobacilli (Piard et al., 1997). The authors attributed the differences in successful anchoring to cell wall composition or cell wall turn over. This finding was reproduced by Dieye et al. (2001) who showed inefficient cell wall localization of their reporter protein, staphylococcal nuclease A, in L. lactis when it was coupled to the M6 protein cell wall anchor and signal peptide. By switching the signal peptide to one of lactococcal origin (Usp45), M6 was able to be efficiently displayed in $L$. lactis as well as in other LAB including L. casei, $L$. sakei, and L. plantarum. These studies suggest that the sortase machinery is functionally different across LAB and have the capacity to recognize substrates from an unrelated microbe (Piard et al., 1997; Dieye et al., 2001).

Demonstration of the cross functionality of LPXTG cell wall anchoring across Gram-positive species pointed to new directions in vaccine design. Localization of antigen to the cell wall had been shown to not only be effective, but also substantially increases immune responses compared to the intracellular or secreted form of the antigen (Reveneau et al., 2002). In an initial study of the effectiveness of vaccine delivery of the TTFC in L. lactis, anchoring of TTFC using the PrtP cell wall anchor was found to elicit the most robust anti-toxin immunoglobulin $\mathrm{G}(\mathrm{IgG})$ response as compared to the toxin expressed in the soluble form in a murine model (Norton et al., 1996). In this case, the LPXTG anchor from the PrtP gene used to couple the TTFC antigen to the cell wall was of endogenous origin, as opposed to an exogenous anchoring motif from S. pyogenes used to display the M6 protein in L. lactis. The enhanced immune response against TTFC can be attributed to successful localization of the antigen to the cell wall due to sortase specificity for the endogenous anchor.

Two oncogenes, the E7 antigen from HPV type-16 and the oncofetal antigen, have also been expressed in LAB as potential vaccine candidates (Bermudez-Humaran et al., 2003; Cortes-Perez et al., 2003, 2005; Fredriksen et al., 2010). The E7 antigen is specific to cervical cancer, while the oncofetal antigen has been found on all mammalian tumors (Fredriksen et al., 2010). In both studies, the probiotic LAB species, $L$. plantarum, was used as a model vaccine vector. As was shown in display of TTFC, successful presentation of both the E7 and oncofetal antigen was achieved using a speciesspecific cell wall anchor. The consensus sequence for cell wall anchoring by sortase in L. plantarum differs from that of LPXTG found in lactococci and streptococci as it has been shown to recognize the motif, LPQTXE (Kleerebezem et al., 2003). The use of this $L$. plantarum sortase consensus sequence did not only enhance the efficiency of surface display as indicated by Western blot, but also, in the case of oncofetal antigen, promoted IgG oncofetal-specific immune responses in mice after oral immunization (Fredriksen et al., 2010).

One issue arising from studies of the efficacy of oral administration vaccines, is that differences in $\operatorname{IgG}$ responses to the specific antigen were lower when lactobacilli were fed orally as opposed to administered through the nasal route (Reveneau et al., 2002). Kajikawa et al. (2011) examined this phenomenon when they engineered a recombinant strain of $L$. acidophilus NCFM expressing the Salmonella flagellin (FliC), which was covalently linked to the cell wall using an LPXTG motif. The goal of the study was to evaluate the potential of FliC to serve as a vaccine adjuvant for LAB vaccines; however, it can be argued that the major finding of this research was that antigen display on the cell surface is susceptible to degradation by gastric juices. In order to protect the FliC fragment on the surface of $L$. acidophilus, the recombinant $L$. acidophilus cell suspensions were supplemented with either sodium bicarbonate or soybean trypsin inhibitor (SBTI). Both of these treatments were found to protect the antigen from degradation when incubated in simulated gastric juices; however, SBTI has a greater protective effect likely due to its sequestration of bile away from the bacterial cells thus contributing to increase viability and robust antigen production (Kajikawa et al., 2011).

Taken together these studies indicate that vaccine delivery in LAB using LPXTG or LPXTG-like cell wall anchors has great potential. Additionally, these studies highlight some important considerations in the development of $\mathrm{LAB}$ vaccine vectors. Although cell wall anchoring and surface display functions vary across Gram-positive species, they also indicate sortase specificity 
for its target domain, which leads to differences in the efficiency of antigen display. The study conducted by Kajikawa et al. (2011) indicates that despite efficient surface display, protection of antigens from GIT juices may be crucial in achieving the most robust immune response. Finally, these studies further validated the presence of functional sortase proteins in $\mathrm{LAB}$ which operate in protein anchoring to the cell wall.

\section{CONCLUSION}

Interest in sortase and sortase protein substrates have extended beyond the arena of pathogens and promotion of intimate associations and infection. Rather, sortase is clearly an important mechanism for display of cell surface proteins, a significant niche related trait of commensal and probiotic microbes associated with the intestinal mucosa. It is not difficult to envision further use of sortase machinery present in LAB to present cell surface oral vaccines, given the success of antigen display discussed in this review. The hypothesis that sortase enzymes may play crucial

\section{REFERENCES}

Altermann, E., Russell, W. M., AzcaratePeril, M. A., Barrangou, R., Buck, B. L., Mcauliffe, O., et al. (2005). Complete genome sequence of the probiotic lactic acid bacterium $\mathrm{Lac}$ tobacillus acidophilus NCFM. Proc. Natl. Acad. Sci. U.S.A. 102, 39063912.

Barocchi, M. A., Ries, J., Zogaj, X., Hemsley, C., Albiger, B., Kanth, A., et al. (2006). A pneumococcal pilus influences virulence and host inflammatory responses. Proc. Natl. Acad. Sci. U.S.A. 103, 2857-2862.

Bermudez-Humaran, L. G., CortesPerez, N. G., Le Loir, Y., Gruss, A., Rodriguez-Padilla, C., SaucedoCardenas, O., et al. (2003). Fusion to a carrier protein and a synthetic propeptide enhances E7 HPV-16 production and secretion in Lactococcus lactis. Biotechnol. Prog. 19, 11011104.

Bierne, H., Mazmanian, S. K., Trost, M., Pucciarelli, M. G., Liu, G., Dehoux, P., et al. (2002). Inactivation of the srtA gene in Listeria monocytogenes inhibits anchoring of surface proteins and affects virulence. Mol. Microbiol. 43, 869-881.

Boekhorst, J., De Been, M. W. H. J., Kleerebezem, M., and Siezen, R. J. (2005). Genome-wide detection and analysis of cell wall-bound proteins with LPxTG-like sorting motifs. J. Bacteriol. 187, 4928-4934.

Bolotin, A., Quinquis, B., Renault, P., Sorokin, A., Ehrlich, S. D., Kulakauskas, S., et al. (2004). Complete sequence and comparative genome analysis of the dairy bacterium Streptococcus thermophilus. Nat. Biotechnol. 22, 1554-1558.

Buck, B. L., Altermann, E., Svingerud, T., and Klaenhammer, T. R. (2005).
Functional analysis of putative adhesion factors in Lactobacillus acidophilus NCFM. Appl. Environ. Microbiol. 71, 8344-8351.

Cabanes, D., Dehoux, P., Dussurget, O., Frangeul, L., and Cossart, P. (2002). Surface proteins and the pathogenic potential of Listeria monocytogenes. Trends Microbiol. 10, 238-245.

Clancy, K. W., Melvin, J. A., and McCafferty, D. G. (2010). Sortase transpeptidases: insights into mechanism, substrate specificity, and inhibition. Biopolymers 94, 385-396.

Claesson, M. J., Li, Y., Leahy, S. Canchaya, C., Van Pijkeren, J. P., Cerdeno-Tarraga, A. M., et al. (2006). Multireplicon genome architecture of Lactobacillus salivarius. Proc. Natl. Acad. Sci. U.S.A. 103, 67186723.

Cortes-Perez, N. G., Azevedo, V., Alcocer-Gonzalez, J. M., RodriguezPadilla, C., Tamez-Guerra, R. S., Corthier, G., et al. (2005). Cell-surface display of E7 antigen from human papillomavirus type-16 in Lactococcus lactis and in Lactobacillus plantarum using a new cell-wall anchor from lactobacilli. J. Drug Target. 13, 89-98.

Cortes-Perez, N. G., BermudezHumaran, L. G., Le Loir, Y., Rodriguez-Padilla, C., Gruss, A. Saucedo-Cardenas, O., et al. (2003). Mice immunization with live lactococci displaying a surface anchored HPV-16 E7 oncoprotein. FEMS Microbiol. Lett. 229, 37-42.

Danne, C., and Dramsi, S. (2012). Pili of Gram-positive bacteria: roles in host colonization. Res. Microbiol. $163,645-658$.

Denou, E., Pridmore, R. D., Berger, B., Panoff, J. M., Arigoni, F., and Brussow, H. (2008). Identification of

roles in bacterial physiology (as in the case of PrtP in L. lactis ssp. cremoris MG1363) as well as mediating bacterial-host interactions has accelerated the study of this enzyme in different species of LAB. The ability to access and examine sortase enzymes and their targets using genomic analysis tools has been crucial. The mechanisms of sortase action and covalent linkage of SDPs to the cell wall is a successful tactic of surface display in Gram-positive bacteria which has enabled some pathogenic organisms to gain advantage of their host, while allowing others, namely commensal and probiotic bacteria, to adhere and interact with their hosts in positive way.

\section{ACKNOWLEDGMENTS}

Work on LAB and probiotics in the Klaenhammer group is supported by the North Carolina Dairy Foundation, Danisco/Dupont Nutrition and Health, and Dairy Management, Inc. We would like to thank Dr. Yong Jun Goh and Dr. Sarah O'Flaherty for helpful discussions and manuscript review.

genes associated with the long-gutpersistence phenotype of the probiotic Lactobacillus johnsonii strain NCC533 using a combination of genomics and transcriptome analysis. J. Bacteriol. 190, 3161-3168.

Dieye, Y., Oxaran, V., Ledue-Clier, F., Alkhalaf, W., Buist, G., Juillard, V., et al. (2010). Functionality of sortase A in Lactococcus lactis. Appl. Environ. Microbiol. 76, 7332-7337.

Dieye, Y., Usai, S., Clier, F., Gruss, A., and Piard, J. C. (2001). Design of a protein-targeting system for lactic acid bacteria. J. Bacteriol. 183, 4157-4166.

Edelman, S. M., Lehti, T. A., Kainulainen, V., Antikainen, J., Kylvaja, R., Baumann, M., et al. (2012). Identification of a high-molecular-mass $\mathrm{Lac}$ tobacillus epithelium adhesin (LEA) of Lactobacillus crispatus ST1 that binds to stratified squamous epithelium. Microbiology 158, 17131722.

Ellen, R. P., Walker, D. L., and Chan, K. H. (1978). Association of long surface appendages with adherencerelated functions of the gram-positive species Actinomyces naeslundii. J. Bacteriol. 134, 1171-1175.

Fredriksen, L., Mathiesen, G., Sioud, M., and Eijsink, V. G. (2010). Cell wall anchoring of the 37-kilodalton oncofetal antigen by Lactobacillus plantarum for mucosal cancer vaccine delivery. Appl. Environ. Microbiol. 76, 7359-7362.

Garandeau, C. (2002). The sortase SrtA of Listeria monocytogenes is involved in processing of internalin and in virulence. Infect. Immun. 70, $1382-$ 1390.

Gaspar, A. H., and Ton-That, H. (2006). Assembly of distinct pilus structures on the surface of Corynebacterium diphtheriae. J. Bacteriol. 188, 15261533.

Godon, J. J., Pillidge, C. J., Jury, K., Shearman, C. A., and Gasson, M. J. (1995). Molecular analysis of the Lactococcus lactis sex factor. Dev. Biol. Stand. 85, 423-430.

Goh, Y. J., Goin, C., O’Flaherty, S., Altermann, E., and Hutkins, R. (2011). Specialized adaptation of a lactic acid bacterium to the milk environment: the comparative genomics of Streptococcus thermophilus LMD-9. Microb. Cell Fact. 10:S22. doi: 10.1186/14752859-10-S1-S22

Gross, G., Van Der Meulen, J., Snel, J., Van Der Meer, R., Kleerebezem, M., Niewold, T. A., et al. (2008). Mannose-specific interaction of Lactobacillus plantarum with porcine jejunal epithelium. FEMS Immunol. Med. Microbiol. 54, 215-223.

Hendrickx, A. P., Budzik, J. M., Oh, S. Y., and Schneewind, O. (2011). Architects at the bacterial surface - sortases and the assembly of pili with isopeptide bonds. Nat. Rev. Microbiol. 9, 166-176.

Hols, P., Hancy, F., Fontaine, L., Grossiord, B., Prozzi, D., LeblondBourget, N., etal. (2005). New insights in the molecular biology and physiology of Streptococcus thermophilus revealed by comparative genomics. FEMS Microbiol. Rev. 29, 435-463.

Jacobsen, C. N., Nielsen, V. R., Hayford, A. E., Moller, P. L., Michaelsen, K. F., Paerregaard, A., et al. (1999). Screening of probiotic activities of forty-seven strains of Lactobacillus spp. by in vitro techniques and evaluation of the colonization ability of five selected strains in humans. Appl. Environ. Microbiol. 65, 49494956. 
Kajikawa, A., Nordone, S. K., Zhang, L., Stoeker, L. L., Lavoy, A. S., Klaenhammer, T. R., et al. (2011). Dissimilar properties of two recombinant Lactobacillus acidophilus strains displaying Salmonella FliC with different anchoring motifs. Appl. Environ. Microbiol. 77, 6587-6596.

Kankainen, M., Paulin, L., Tynkkynen, S., Von Ossowski, I., Reunanen, J., Partanen, P., et al. (2009). Comparative genomic analysis of Lactobacillus rhamnosus GG reveals pili containing a human-mucus binding protein. Proc. Natl. Acad. Sci. U.S.A. 106, 17193-17198.

Kleerebezem, M., Boekhorst, J., Van Kranenburg, R., Molenaar, D., Kuipers, O. P., Leer, R., et al. (2003). Complete genome sequence of $\mathrm{Lac}$ tobacillus plantarum WCFS1. Proc. Natl. Acad. Sci. U.S.A. 100, 19901995.

Kleerebezem, M., Hols, P., Bernard, E., Rolain, T., Zhou, M., Siezen, R. J., etal. (2010). The extracellular biology of the lactobacilli. FEMS Microbiol. Rev. 34, 199-230.

Makarova, K., Slesarev, A., Wolf, Y., Sorokin, A., Mirkin, B., Koonin, E., et al. (2006). Comparative genomics of the lactic acid bacteria. Proc. Natl. Acad. Sci. U.S.A. 103, 15611-15616.

Maresso, A. W., and Schneewind, O. (2008). Sortase as a target of antiinfective therapy. Pharmacol. Rev. 60, 128-141.

Marraffini, L. A., Dedent, A. C., and Schneewind, O. (2006). Sortases and the art of anchoring proteins to the envelopes of gram-positive bacteria. Microbiol. Mol. Biol. Rev. 70, 192-221.

Mazmanian, S. K., Liu, G., Jensen, E. R., Lenoy, E., and Schneewind, O (2000). Staphylococcus aureus sortase mutants defective in the display of surface proteins and in the pathogenesis of animal infections. Proc. Natl. Acad. Sci. U.S.A. 97, 5510-5515.

Mishra, A., Das, A., Cisar, J. O., and TonThat, H. (2007). Sortase-catalyzed assembly of distinct heteromeric fimbriae in Actinomyces naeslundii. J. Bacteriol. 189, 3156-3165.

Mora, M., Bensi, G., Capo, S., Falugi, F., Zingaretti, C., Manetti, A. G. O., et al. (2005). Group A Streptococcus produce pilus-like structures containing protective antigens and Lancefield T antigens. Proc. Natl. Acad. Sci. U.S.A. 102, 15641-15646.
Moran, A. P., Gupta, A., and Joshi, L. (2011). Sweet-talk: role of host glycosylation in bacterial pathogenesis of the gastrointestinal tract. Gut 60, 1412-1425.

Munoz-Provencio, D., Rodriguez-Diaz, J., Collado, M. C., Langella, P., Bermudez-Humaran, L. G., and Monedero, V. (2012). Functional analysis of the Lactobacillus case BL23 sortases. Appl. Environ. Microbiol. 78, 8684-8693.

Norton, P. M., Brown, H. W. G., Wells, J. M., Macpherson, A. M., Wilson, P. W. and Le Page, R. W. F. (1996). Factors affects the immunogenicity of tetanus toxin fragment $\mathrm{C}$ expressed in $\mathrm{Lac}$ tococcus lactis. FEMS Immunol. Med. Microbiol. 14, 167-177.

O'Callaghan, J., Butto, L. F., Macsharry, J., Nally, K., and O'Toole, P. W. (2012). Influence of adhesion and bacteriocin production by Lactobacillus salivarius on the intestinal epithelial cell transcriptional response. Appl. Environ. Microbiol. 78, 5196-5203.

O'Flaherty, S., and Klaenhammer, T. R. (2012). Influence of exposure time on gene expression by human intestinal epithelial cells exposed to Lactobacillus acidophilus. Appl. Environ. Microbiol. 78, 5028-5032.

Oxaran, V., Ledue-Clier, F., Dieye, Y., Herry, J. M., Pechoux, C., Meylheuc, T., et al. (2012). Pilus biogenesis in Lactococcus lactis: molecular characterization and role in aggregation and biofilm formation. PLoS ONE 7:e50989. doi: 10.1371/journal.pone.0050989

Piard, J. C., Hautefort, I., Fischetti, V. A., Ehrlich, S. D., Fons, M., and Gruss, A. (1997). Cell wall anchoring of the Streptococcus pyogenes M6 protein in various lactic acid bacteria. J. Bacteriol. 179, 3068-3072.

Pinto, M. G. V., Gomez, M. R., Seifert, S., Watzl, B., Holzapfel, W. H. and Franz, C. M. (2009). Lactobacilli stimulate the innate immune response and modulate the TLR expression of HT29 intestinal epithelial cells in vitro. Int. J. Food Microbiol. 133, 86-93.

Remus, D. M., Bongers, R. S., Meijerink, M., Fusetti, F., Poolman, B., De Vos, P., et al. (2013). The impact of Lactobacillus plantarum sortase on target-protein sorting, gastrointestinal persistence, and host immune response modulation. J. Bacteriol. 195, 502-509.
Reunanen, J., Von Ossowski, I., Hendrickx, A. P., Palva, A., and De Vos, W. M. (2012). Characterization of the SpaCBA pilus fibers in the probiotic Lactobacillus rhamnosus GG. Appl. Environ. Microbiol. 78, 2337 2344.

Reveneau, N., Geoffroy, M. C., Locht, C., Chagnaud, P., and Mercenier, A. (2002). Comparison of the immune responses induced by local immunizations with recombinant Lactobacillus plantarum producing tetanus toxin fragment $\mathrm{C}$ in different cellular locations. Vaccine 20, 1769 1777.

Roos, S., and Jonsson, H. (2002). A high-molecular-mass cell-surface protein from Lactobacillus reuteri 1063 adheres to mucus components. Microbiology 148, 433-442.

Schaffer, C., and Messner, P. (2005). The structure of secondary cell wall polymers: how Gram-positive bacteria stick their cell walls together. Microbiology 151, 643-651.

Shirazi, T., Longman, R. J., Corfield, A. P., and Probert, C. S. (2000). Mucins and inflammatory bowel disease. Postgrad. Med. J. 76, 473-478.

Spirig, T., Weiner, E. M., and Clubb, R. T. (2011). Sortase enzymes in Grampositive bacteria. Mol. Microbiol. 82, 1044-1059.

Stalhammar-Carlemalm, M., Areschoug, T., Larsson, C., and Lindahl, G. (1999). The R28 protein of Streptococcus pyogenes is related to several group B streptococcal surface proteins, confers protective immunity and promotes binding to human epithelial cells. Mol. Microbiol. 33, 208-219.

Telford, J. L., Barocchi, M. A., Margarit, I., Rappuoli, R., and Grandi, G. (2006). Pili in gram-positive pathogens. Nat. Rev. Microbiol. 4, 509-519.

Ton-That, H., and Schneewind, O. (2003). Assembly of pili on the surface of Corynebacterium diphtheriae. Mol. Microbiol. 50, 1429-1438.

van Pijkeren, J. P., Canchaya, C., Ryan, K. A., Li, Y., Claesson, M. J., Sheil, B., et al. (2006). Comparative and functional analysis of sortase-dependent proteins in the predicted secretome of Lactobacillus salivarius UCC118. Appl. Environ. Microbiol. 72, 4143 4153.

Ventura, M., O’Flaherty, S., Claesson, M. J., Turroni, F., Klaenhammer,
T. R., Van Sinderen, D., et al. (2009). Genome-scale analyses of health-promoting bacteria: probiogenomics. Nat. Rev. Microbiol. 7, 61-71.

von Ossowski, I., Reunanen, J., Satokari, R., Vesterlund, S., Kankainen, M., Huhtinen, H., et al. (2010). Mucosal adhesion properties of the probiotic Lactobacillus rhamnosus GG SpaCBA and SpaFED pilin subunits. Appl. Environ. Microbiol. 76, 20492057.

Vos, P., Simons, G., Siezen, R. J., and Devos, W. M. (1989). Primary structure and organization of the gene for a procaryotic, cell envelope-located serine proteinase. J. Biol. Chem. 264, 13579-13585.

Weidenmaier, C., and Peschel, A. (2008). Teichoic acids and related cell-wall glycopolymers in Grampositive physiology and host interactions. Nat. Rev. Microbiol. 6, 276-287.

Zhou, M., Theunissen, D., Wels, M., and Siezen, R. J. (2010). LAB-Secretome: a genome-scale comparative analysis of the predicted extracellular and surface-associated proteins of Lactic Acid Bacteria. BMC Genomics 11:651. doi: 10.1186/1471-2164-11-651

Conflict of Interest Statement: The authors declare that the research was conducted in the absence of any commercial or financial relationships that could be construed as a potential conflict of interest.

Received: 06 February 2013; paper pending published: 03 March 2013; accepted: 14 March 2013; published online: 08 April 2013.

Citation: Call EK and Klaenhammer TR (2013) Relevance and application of sortase and sortase-dependent proteins in lactic acid bacteria. Front. Microbiol. 4:73. doi: 10.3389/fmicb.2013.00073 This article was submitted to Frontiers in Food Microbiology, a specialty of Frontiers in Microbiology.

Copyright (c) 2013 Call and Klaenhammer. This is an open-access article distributed under the terms of the Creative Commons Attribution License, which permits use, distribution and reproduction in other forums, provided the original authors and source are credited and subject to any copyright notices concerning any third-party graphics etc. 